\title{
Islet-specific Prmt5 excision leads to reduced insulin expression and glucose intolerance in mice
}

\author{
Jian Ma1,2,*, Xin He1,*, Yan Cao1,2, Kienan O'Dwyer¹, Katherine M Szigety1, Yuan Wu1, Buddha Gurung1, Zijie Feng1, \\ Bryson W Katona1,3 and Xianxin Hua ${ }^{1,2}$
}

\begin{abstract}
'Department of Cancer Biology, Abramson Family Cancer Research Institute, University of Pennsylvania Perelman School of Medicine, Philadelphia, Pennsylvania, USA

Institute for Diabetes, Obesity, and Metabolism, University of Pennsylvania Perelman School of Medicine, Philadelphia, Pennsylvania, USA

${ }^{3}$ Division of Gastroenterology, University of Pennsylvania Perelman School of Medicine, Philadelphia, Pennsylvania, USA
\end{abstract}

Correspondence should be addressed to X Hua: huax@pennmedicine.upenn.edu

*(J Ma and X He contributed equally to this work)

\begin{abstract}
Protein arginine methyltransferase 5 (PRMT5), a symmetric arginine methyltransferase, regulates cell functions by influencing gene transcription through posttranslational modification of histones and non-histone proteins. PRMT5 interacts with multiple partners including menin, which controls beta cell homeostasis. However, the role of Prmt5 in pancreatic islets, particularly in beta cells, remains unclear. A mouse model with an islet-specific knockout (KO) of the Prmt5 gene was generated, and the influence of the Prmt5 excision on beta cells was investigated via morphologic and functional studies. Beta cell function was evaluated by glucose tolerance test (GTT) and glucose-stimulated insulin secretion (GSIS) test. Beta cell proliferation was evaluated by immunostaining. Gene expression change was determined by real-time qPCR. Molecular mechanisms were investigated in beta cells in vitro and in vivo in Prmt5 KO mice. The results show that islet-specific KO of Prmt5 reduced expression of the insulin gene and impaired glucose tolerance and GSIS in vivo. The mechanistic study indicated that PRMT5 is involved in the regulation of insulin gene transcription, likely via histone methylation-related chromatin remodeling. The reduced expression of insulin in beta cells in the Prmt5 KO mice may contribute to impaired glucose tolerance (IGT) and deficient GSIS in the mouse model. These results will provide new insights into exploring novel strategies to treat diabetes caused by insulin insufficiency.
\end{abstract}
Key Words
- PRMT5
- beta cell
- impaired glucose tolerance
- insulin transcription

\section{Introduction}

Diabetes is a complex and heterogeneous disease accompanied by a deterioration of glycemic control (Ashcroft \& Rorsman 2012, DeFronzo et al. 2015). Dysfunction of insulin-secreting beta cells, such as reduced insulin synthesis, reduces the amount of secreted insulin or diminishes pulsatile secretion of insulin in response to a stimulus, contributing to the initiation of diabetes (Olson et al. 1993, Ashcroft \& Rorsman 2012, Ferrannini \& Mari 2014, Antonioli et al. 2015). Growing evidence suggests that epigenetic mechanisms are involved in regulating 
beta cell dysfunction, beta cell age and the pathological process of diabetes (Pinney \& Simmons 2010, Menzies et al. 2016, Bernstein et al. 2017). Several important epigenetic regulators, including DNA methyltransferases and histone-modifying enzymes (such as DNMT3A, EZH2, and p300), have been shown to regulate beta cell function (Chen et al. 2009, Fernandez-Zapico et al. 2009, Papizan et al. 2011, Dhawan et al. 2015, Rui et al. 2016).

Recently, a methyltransferase, protein arginine methyltransferase5 (PRMT5), was shown to regulate cells fate by diverse epigenetic means via influencing posttranslational modifications (Bedford \& Clarke 2009, Yang \& Bedford 2013). PRMT5, together with its cofactor MEP50, mediates the symmetric dimethylation of histones $\mathrm{H} 2 \mathrm{~A}$ and $\mathrm{H} 4$ at R3 and histone $\mathrm{H} 3$ at R8 (Pal et al. 2007, Girardot et al. 2014). The symmetric dimethylation of these histone residues generally generates repressive marks for gene transcription, and PRMT5 has been found in several transcriptional repressor complexes, including those containing SIN3A/HDAC, MBD2/NURD, and N-CoR/SMRT (Pal et al. 2003, Le Guezennec et al. 2006). Moreover, PRMT5 represses globin gene expression through recruitment of DNMT3A, indicating a potential crosstalk between histone arginine methylation and DNA methylation (Zhao et al. 2009, Rank et al. 2010). In addition to histones, PRMT5 regulates a number of non-histone proteins with functions in RNA processing, translation, signal transduction, apoptosis, and cell cycle regulation (Wei et al. 2014). Unexpectedly, PRMT5 has also been reported to activate the transcription of certain genes that are involved in adipogenic differentiation or skeletal muscle differentiation (Dacwag et al. 2007,
LeBlanc et al. 2012). We previously showed that menin, a crucial epigenetic regulator that controls islet homeostasis (Matkar et al. 2013, Feng et al. 2017a), interacts with PRMT5 to suppress cell proliferation, partly through regulating Hedgehog signaling (Gurung et al. 2013). However, the role of PRMT5 in beta cell proliferation and islet function has not yet been investigated in vivo.

Here, we developed Prmt5 islet-specific conditional knockout (KO) mice and evaluated the impact of Prmt5 KO on islets. Notably, we found that Prmt5 KO impaired glucose tolerance (GT) and glucose stimulation-induced insulin secretion (GSIS). PRMT5 promotes insulin transcription and histone methylation at the insulin gene locus. The reduced insulin expression in beta cells in the Prmt5 KO mice likely contributes to the impaired GT and defective GSIS in the mouse model.

\section{Materials and methods}

\section{Mice and cell line}

Cre/loxP strategy was used to develop Prmt5 isletspecific conditional KO mouse (Fig. 1). The mice with Prmt5tm1a (ЕUСОMM) Wtsi (from the Wellcome Sanger Institute, Cambridge, UK) were bred with FLPeR mice (B6.129S4-Gt (ROSA) 26Sortm1 (FLP1) Dym/Rain J (from the Jackson Laboratory), resulting in excision of the FRT-flanking lacZ and neomycin sequence. Subsequent breeding between F1 mice generated a population of progeny that included the desired Prmt5 $5_{\text {loxP } / o x P(/ /)}$ genotype. The mice with Prmt5// and the mice expressing Pdx1-driven Cre-ER recombinase

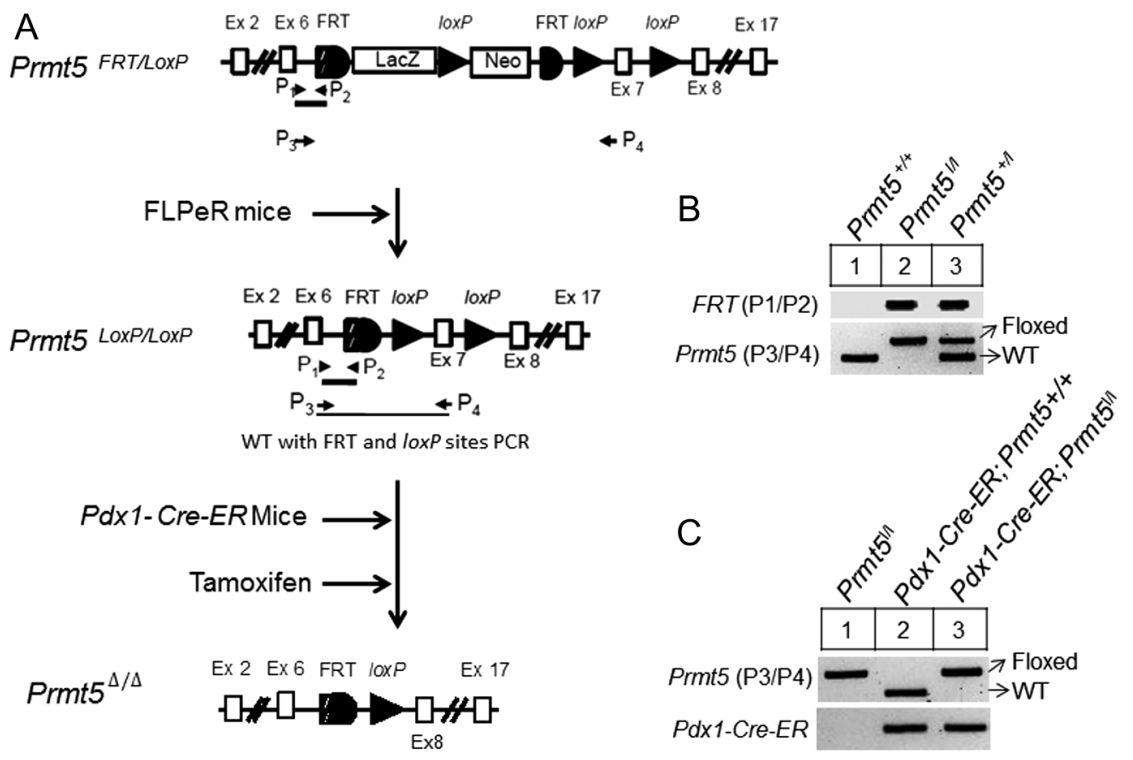

Figure 1

Diagrams of generation of Pdx1-Cre-ER, Prmt5//I mice. (A) The mice with Prmt5tm1a (EUCOMM) Wtsi allele consisting of FRT sites followed by a LacZ sequence and loxP sites, were bred with FLPeR mice (B6.12954-t (ROSA) 26Sortm1 (FLP1) Dym/Rain J), resulting in excision of the FRTflanking lacZ and neomycin sequence. The F1 progeny were heterozygous for Prmt5 (Prmt5+//). Subsequent breeding between F1 mice resulted in a population of progeny that included the desired genotype Prmt5//I. Then, Pdx1-CreER; Prmt5//I mice were generated by intercrossing $P d \times 1$-CreER mice with Prmt5//I mice. (B, C) The genotypes were verified by PCR using tail DNA. 
were intercrossed to generate homozygous $P d x$ 1-CreER; Prmt5//l mice. The mice with $P d x$ 1-CreER genotype was initially obtained the from Dr Melton's group (Gu et al. 2002). They were extensively used in our previously published work and shown tissue-specific and tamoxifen (TAM)-induced Men1 deletion in islets (Yang et al. 2010). The pups from the breeding between Prmt5// and $P d x$ 1-CreER mice were genotyped routinely by PCR using the tail genomic DNA for the floxed Prmt5 and the $P d x 1-C r e-E R$ transgenes.

Pdx 1-CreER; Prmt5// mice and control mice were administrated with TAM (MP Biomedicals, Santa Ana, CA, USA) at $200 \mathrm{mg} / \mathrm{kg}$ of body weight by gavage daily for two consecutive days, followed by 1 day off and then for another two consecutive days (Yang et al. 2010). Male mice with age-matched were used in this study, because male mice are more severely affected by type 2 diabetes than female mice generally. All mice were housed in a pathogen-free environment on a 12-h light/darkness cycle with ad libitum access to water and normal chow diet in the animal facility of University of Pennsylvania. All animal experiments and methods were approved by the Institutional Animal Care and Use Committee of University of Pennsylvania.

Rat insulinoma INS-1 cell line was obtained from Dr Xiaosong Ma (Kong et al. 2014), and the cells (passage 70-80) were maintained in RPMI-1640 medium (4.6 mM glucose), supplemented with $10 \%(\mathrm{v} / \mathrm{v})$ FBS (Sigma-Aldrich), $1 \mathrm{mM}$ sodium pyruvate, $10 \mathrm{mM}$ HEPES, $50 \mu \mathrm{M} \beta$-mercaptoethanol, 100 units $/ \mathrm{mL}$ penicillin and $100 \mu \mathrm{g} / \mathrm{mL}$ streptomycin at $37^{\circ} \mathrm{C}$ and $5 \% \mathrm{CO}_{2}$.

\section{Physiological measurements}

Blood glucose levels were assayed from tail vein blood. Glucose tolerance test (GTT; glucose at $2 \mathrm{~g} / \mathrm{kg}$ of body weight, i.p.) and GSIS (glucose at $3 \mathrm{~g} / \mathrm{kg}$ of body weight, i.p.) were performed on mice fasting overnight (16h) (Yang et al. 2010). In brief, for GTT, the mice were fasted for $16 \mathrm{~h}$ and then injected intraperitoneally (i.p.) with glucose $(2 \mathrm{~g} / \mathrm{kg}$ of body weight). Blood samples at 0,15 , $30,60,90$, and $120 \mathrm{~min}$ after the injection were obtained from tail-tip bleedings, and blood glucose levels were measured with a Glucometer (Lifescan, Wayne, USA). For GSIS, glucose $(3 \mathrm{~g} / \mathrm{kg}$ of body weight) was injected i.p. and blood obtained by submandibular venipuncture was collected at 0, 15, and $30 \mathrm{~min}$ in heparinized tubes. Serum insulin levels were measured by ELISA using a mouse insulin kit (Crystal Chem, Elk Grove Village, IL, USA).

\section{Islet isolation and perifusion}

Islets were isolated by pancreatic digestion, purified using a Ficoll-paque gradient (GE Healthcare). Immediately after collection, the purified islets were used for RNA extraction. For perifusion study, the islets need to be incubated 2 days at $37^{\circ} \mathrm{C}$ with $5 \% \mathrm{CO}_{2}$ before glucose stimulation.

Islets perifusion studies in vitro were carried out by Islet Cell Biology Core at University of Pennsylvania using an automated perifusion system. After 2-day incubation in PRMI1640 medium, islets were transferred into the perifusion system and subjected to 0 , low $(3 \mathrm{mM})$ and high $(16.7 \mathrm{mM})$ glucose concentrations at $0 \mathrm{~min}$, 30th min and 50th $\mathrm{min}$, and then switched back to $0 \mathrm{mM}$ glucose at $70 \mathrm{~min}$, and followed by a KCL $(30 \mathrm{mM})$ stimulation at 90th min for another $20 \mathrm{~min}$. Insulin in the medium was harvested automatically and measured with Radioimmunoassay and Biomarkers Core at University of Pennsylvania using ELISA.

\section{Gene expression analysis by quantitative real-time PCR (qPCR)}

Total RNA was isolated from mouse islets and beta cell lines using RNA Mini Kit (Qiagen) and cDNA prepared was using Superscript III Reverse transcriptase (Invitrogen, Life Technologies) according to manufacturer's instructions. cDNA was used for qPCR with specific primers (Supplementary Table 1, see section on supplementary materials given at the end of this article) in the presence of Power SYBR Master Mix (Qiagen) using 7900HT qPCR machine (Applied Biosystems). All analyses were done in triplicate. Relative mRNA levels were analyzed using $\Delta \Delta \mathrm{Ct}$ method.

\section{Western blot analysis}

Cells were collected and lysed with RIPA lysis buffer (Sigma), followed by centrifugation at $20,000 \boldsymbol{g}$ for $10 \mathrm{~min}$ at $4^{\circ} \mathrm{C}$. Protein concentration was determined by $\mathrm{BCA}$ assay (Sigma-Aldrich). Cellular proteins were separated by 4.0-20.0\% SDS-PAGE (Genscript, Piscataway Township, NJ, USA) and then transferred to a PVDF membrane. The membrane was blocked by TBST with 5\% fat free milk, and incubated with antibodies directed against the following proteins: Insulin (1:1000) (Cell Signaling Technology, Cat \# 8138S); PRMT5 (1:1000) (Cell Signaling Technology, Cat \# ab31751); and $\beta$-actin (1:10000) (Sigma-Aldrich, Cat \# A5441). Immunoreactive proteins were visualized by 
exposure to X-ray film. Protein bands were analyzed by image scanning.

\section{Chromatin immunoprecipitation (ChIP)}

INS- 1 cells were plated at a density of $2 \times 10^{6}$ cells $/ 10 \mathrm{~cm}$ tissue culture plate, and cultured for $36 \mathrm{~h}$ with each treatment condition. After collection of cells, ChIP assays were performed according to the manufacturer's instructions using a QuickChIP kit (Novus Biologicals, Centennial, CO, USA), as previously reported (Matkar et al. 2015, Feng et al. $2017 b$ ). Briefly, cells were fixed with $1 \%$ formaldehyde and then lysed according to the protocol in a ChIP lysis buffer with protease inhibitors, and cellular DNA was sheared with sonication. After clearing, this lysate was incubated with either control IgG or a specific primary antibody $(4 \mu \mathrm{g})$ at $4^{\circ} \mathrm{C}$ overnight, and collected with protein $\mathrm{A} / \mathrm{G}$ agarose beads. The antibodies used here include anti-PRMT5 (Cell Signaling Technology, Cat \# ab31751), anti-BRG1 (Abcam Cat \# ab110641), anti-H3R8 (Abcam, Cat \# ab130740), and anti-IgG (Abcam, Cat \# ab46540). The protein-DNA complexes were eluted from the beads and DNA was amplified by qPCR using primer pairs (Supplementary Table 1) specific to the insulin promoter. Reactions were done in duplicate, and results are normalized to input chromatin and are reported as $\%$ input \pm s.D.

\section{ShRNA, Lentivirus packaging and infection}

Lentiviral packing plasmids pMD2.G and psPAX2 were purchased from Sigma-Aldrich. shRNA plasmids for Prmt5 was pLKO.1-puromycin backbone-based and obtained from the University of Pennsylvania Perelman School of Medicine High-Throughput Screening Core. To produce lentivirus, as previously reported (Matkar et al. 2015), 293T cells were transfected with pMD2.G, psPAX2, and the plasmid of Prmt5 shRNA using Fugene 6 (Promega) according to the manufacturer's instructions. For all virus production, after collecting and filtering the virus, cells were then transduced. Twenty-four hours after completion of transduction, cells were then selected with puromycin for $72 \mathrm{~h}$.

\section{Immunofluorescence (IF) and morphometric analysis}

Mouse whole pancreas tissue was fixed in $4 \%$ formalin and processed for paraffin embedding. Antigen retrieval was performed by microwave heating in antigen retrieval solution-citrate buffered (Abcam). Detection was done using fluorochrome-conjugated secondary antibodies (Becton Dickenson) according to manufacturer's instructions as previously reported (Yang et al. 2010). The following primary antibodies were used: anti-Insulin (1:200) (Cell Signaling Technology, Cat \# 8138S), antiPrmt5 (1:200) (Cell Signaling Technology, Cat \# ab31751), and anti-Ki67 (1:200) (Abcam, Cat \#ab15580). Cell nuclear staining was performed using DAPI (Sigma-Aldrich, Cat \# 10236276001). The sections were visualized using a Nikon Eclipse E800 fluorescent microscope with a CCD digital camera. Whole pancreas scanning was implemented using a Leica DMI6000B fluorescent microscope. Quantification of insulin-positive areas and staining intensity was performed with ImageJ software.

\section{Statistical analysis}

Significant differences between two groups were determined by Tukey's test. The ANOVA method was used for statistical analysis on multiple groups. Analysis was performed using the Prism GraphPad v4.0 (GraphPad Software Inc.). Values are expressed as mean \pm s.D., of which a representative experiment is depicted in the figures. $P$ values $<0.05$ were considered statistically significant.

\section{Results}

\section{Generation of Lox-P-mediated Prmt5 islet-specific conditional knockout (KO) mouse model}

To generate the mouse strains in which we can conditionally delete the Prmt5 gene in islets in a time or tissue-specific manner, the mice with Prmt $5^{1 / 1}$ genotype were crossed with mice expressing Pdx1-driven Cre-ER, a fusion protein between CRE (loxP site-specific DNA recombinase) and part of the estrogen receptor, the fusion protein can cleave the loxP sites specifically when 4-hydroxy tamoxifen (TAM) binds the ER moiety of CRE-ER, as previously described (Yang et al. 2010) (Fig. 1A). Three groups of mice with genotypes including Pdx1-Cre-ER; Prmt51/1, Prmt5// (Control1), and Pdx1-Cre-ER; Prmt5 $^{+l+}$ (Control2) were identified using PCR (Fig. 1C). Then, these mice were fed with TAM, and Prmt5 excision in pancreatic islets was determined by qPCR and immunostaining of PRMT5.

\section{The islet-specific conditional KO of Prmt5 leads to IGT and defective GSIS}

PDX1 expression was shown to be generally limited to pancreatic islets and duodenal epithelium in the adult, 
especially in beta cells and some delta cells (Miller et al. 1994, Peers et al. 1994, Peshavaria et al. 1994, Guz et al. 1995, Wu et al. 1997, Fu et al. 2013). Thus, the deletion of Prmt 5 induced by Pdx1-driven Cre-ER is supposed to occur in mouse islets. We set up three groups of mice: Prmt51/l (Control1), Pdx1-Cre-ER; Prmt5 ${ }^{+l+}$ (Control2), and Pdx1-Cre-ER; Prmt5//, started to gavage them with TAM, and monitored the blood glucose level as shown in Fig. 2A. After TAM treatment, the Prmt5/// (Control1) mice were designated as Prmt5 ${ }^{1 /}$, the Pdx1-Cre-ER; Prmt5 ${ }^{+l+}$ (Control2) mice were designated as Cre-ER, and Pdx1-Cre-ER; Prmt5// mice were designated as $P r m t 5^{\Delta / \Delta}$, respectively. The bodyweight of the three groups of mice was measured weekly up to the ninth week post TAM administration, and no obvious difference in body weight was observed among the groups during the period of time (Fig. 2B). Histological examination of PRMT5 expression level in pancreas sections from the three different groups of mice using IF staining indicates an obvious deletion of PRMT5 in islets of the Prmt5 $5^{\Delta / \Delta}$ mice compared to those in the other two groups of control mice (Fig. 2C). The staining result of PRMT5 on pancreas section from Pdx1-Cre-ER; Prmt5//l mice without TAM administration is similar with the PRMT5 staining of two control group mice (data not show), indicating no leaky effect of Pdx1driven Cre-ER on Prmt5 excision. Consistently, PRMT5 mRNA level in purified mouse islets also showed a significant decrease in islets of the Prmt5 $\Delta / \Delta$ mice as compared to that in control mice (Fig. 2D). Certain level $(\sim 20 \%)$ of the remaining Prmt5 mRNA in islets from Prmt5 $5^{\Delta / \Delta}$ mice was still detectable by qPCR, suggesting that in some islet cells from mice treated with TAM may still have one or two alleles of the floxed Prmt5. Nevertheless, these results indicate that the Pdx-1-Cre-ER system we used here is a TAM inducible and effective to Prmt5 excision.

To determine the effect of Prmt5 KO on beta cell function in vivo, we performed the glucose tolerance test (GTT) on the mice at week 3, 6, and 9 post TAM administration. The result showed that no obvious change in glucose tolerance (GT) was observed among the three groups 3 weeks after TAM treatment. However, GT was gradually impaired in Prmt $5^{\Delta / \Delta}$ mice from 6 to 9 weeks after Prmt5 excision (Fig. 3A, B and C). Approximately two-fold increase in area under curve (AUC) was observed in Prmt5 KO mice as compared to control mice at ninth week (Fig. 3C). In addition, fasting blood glucose level of the Prmt $5^{\Delta / \Delta}$ group was only moderately higher than that in the control groups at ninth week (Fig. 3D), whereas random blood glucose number of the Prmt $5^{\Delta / \Delta}$ group of mice was significantly higher than other groups of mice (Fig. 3E). To further examine whether PRMT5 deletion affects the GSIS, the above three groups of mice were injected with glucose, followed by detection of the blood insulin level. The results indicate that the control mice (i.e. Prmt5//l and Cre-ER) showed enhanced level of serum insulin following glucose uptake (Fig. 3F). However, Prmt5 KO mice $\left(\mathrm{Prmt5}^{\Delta / \Delta}\right)$ showed markedly defective GSIS (Fig. 3F), concomitant with the marked IGT (Fig. 3B and C).

To further clarify the time course of the defect observed in Prmt5 KO mice involving impaired insulin secretion, in vitro perifusion studies were performed in Prmt5 KO and control islets isolated at the first and sixth weeks post TAM administration (Fig. 4A). The isolated islets were also used to extract RNA and do qPCR to test mRNA
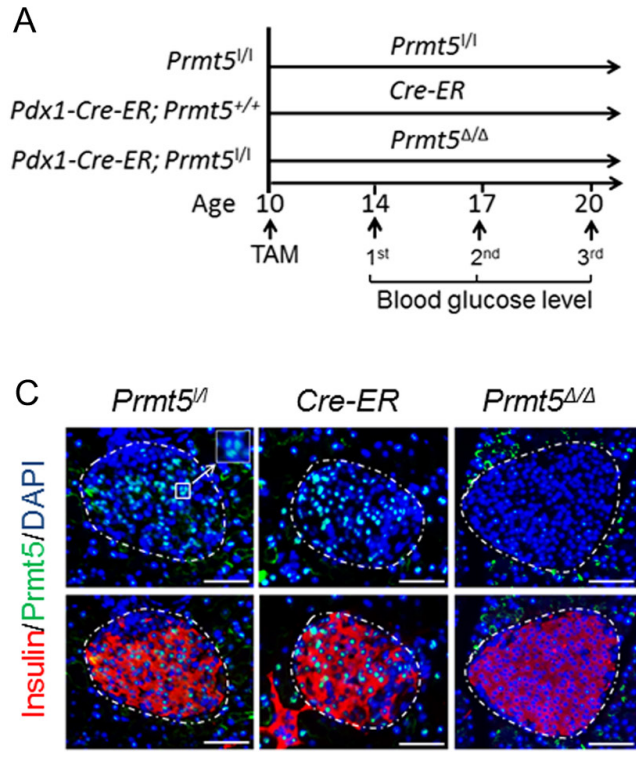

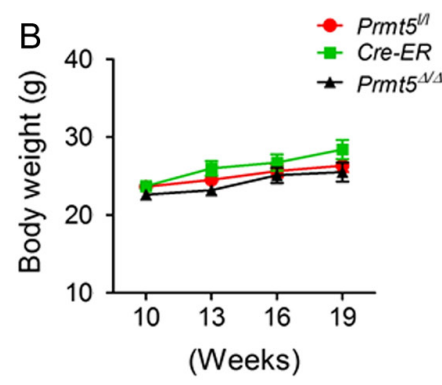

D

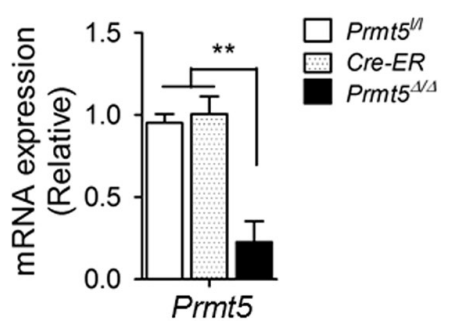

Figure 2

Acute Prmt5 excision in mice islets was induced by TAM treatment. (A) A schema of experimental design. Prmt5"/, Pdx1-Cre-ER; Prmt5 +/+, and $P d x-1-C r e-E R ;$ Prmt5/I mice (male mice, $n=5$ for each group) were fed TAM at the age of 10 weeks. After TAM treatment, the Prmt5//I (Control1) mice were designated as Prmt5/I, the Pdx1-Cre-ER; Prmt5 ${ }^{+/+}$(Control2) mice were designated as Cre-ER, and Pdx1-Cre-ER; Prmt5//I mice were designated as $P r m t 5 \Delta / \Delta$, respectively. Mice were euthanized at 10 th week after TAM feeding. GTT were done before and at third, sixth, and ninth week post TAM treatment. (B) Body weight change for all experimental group mice after TAM treatment. (C) Immunostaining for PRMT5 and insulin on pancreas samples of three different groups of mice. Scale bar, $25 \mu \mathrm{m}$. (D) Comparison of PRMT5 mRNA level between isolated islets of control mice (Prmt5//I and Cre-ER) and Prmt5 excision mice $(P r m t 5 \Delta / \Delta)$. ${ }^{*} P<0.01$. https://joe.bioscientifica.com https://doi.org/10.1530/JOE-19-0268 (c) 2019 Society for Endocrinology Published by Bioscientifica Ltd. Printed in Great Britain 

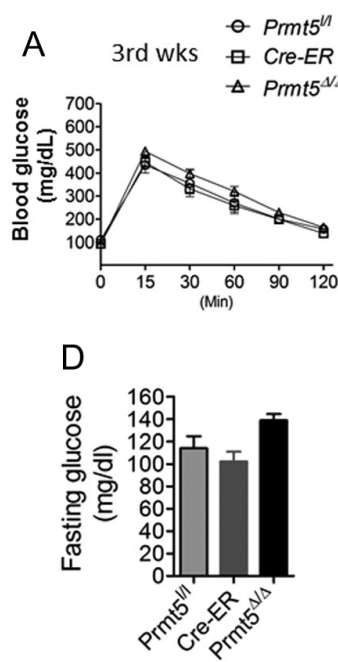
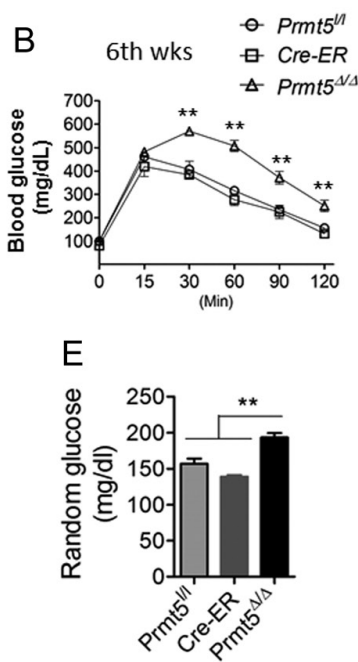

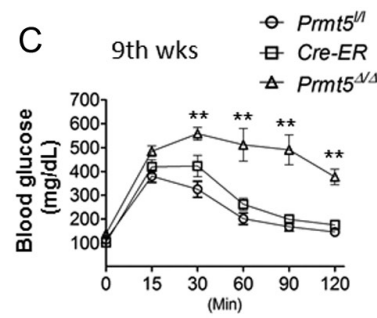

$\mathrm{F}$

- $\operatorname{Prmt5}^{n}$
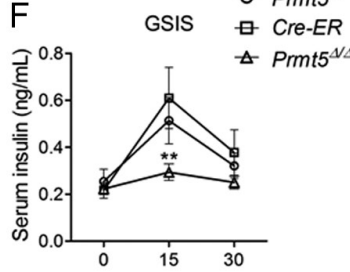

Figure 3

Islet-specific Prmt5 KO in mice impaired GT and GSIS. (A, B, C) Glucose tolerance test (GTT, glucose at $2 \mathrm{~g} / \mathrm{kg}$ of body weight, i.p.). All mice tested in experiment have been starved overnight (16 h) at third, sixth, and ninth week post TAM treatment ( $n=5$ for each group). (D, E) Fasting glucose number and random glucose number were tested on the three groups of the mice at ninth week post TAM administration. (F) In vivo glucosestimulated insulin secretion (GSIS, glucose at $3 \mathrm{~g} / \mathrm{kg}$ of body weight, i.p. $n=5$ for each group) test. All mice tested in experiment have been starved overnight (16h) at 10th week after TAM treatment. $* * P<0.01$ level of PRMT5 and insulin. The results showed that the expression of Prmt5 was reduced to about 50\% relative to control mice at the first week after TAM treatment (Fig. 4B). Unexpectedly, insulin mRNA level was declined by approximately 50\% compared to control mice (Fig. 4C). For the perifusion results, insulin release in response to $16.8 \mathrm{mM}$ glucose was markedly reduced in Prmt5 KO mice (Fig. 4D). For the islets isolated from the mice at sixth week post TAM administration, the mRNA level of PRMT5 and insulin decreased even further to about 25\% of the control (Fig. 4E and F). The insulin secretion was also obviously reduced as compared with the control mice (Fig. 4G).

As Prmt5 KO led to significant reduction of insulin synthesis in beta cells, one may expect to observe marked IGT and quite low fasting insulin level.

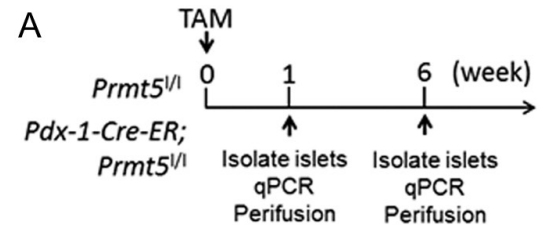

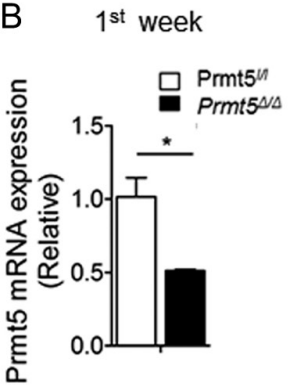

C $1^{\text {st }}$ week
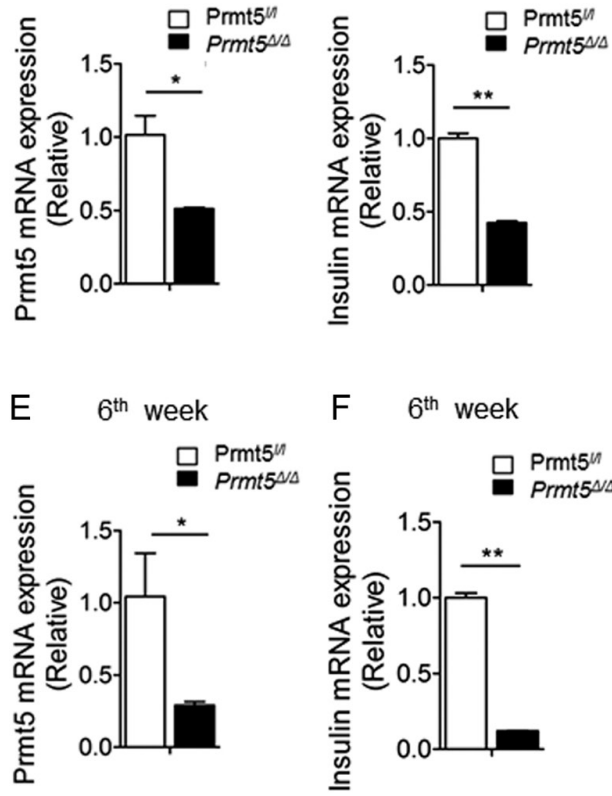

F $\quad 6^{\text {th }}$ week
D $1^{\text {st }}$ week

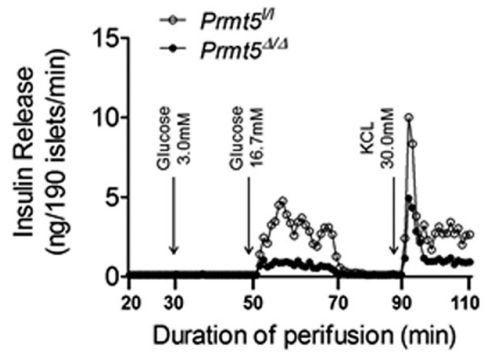

G $\quad 6^{\text {th }}$ week

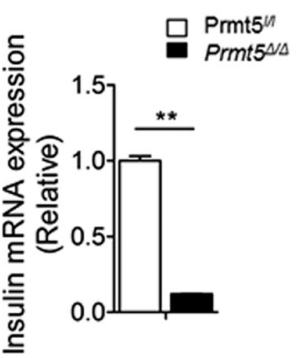

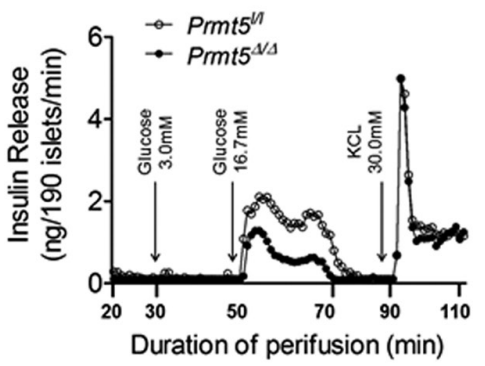

\section{Figure 4}

Islet-specific Prmt5 KO in mice downregulated the insulin expression in islets and impaired the glucose-stimulated insulin release in perifusion studies. (A) schema of experimental design. Prmt5/I and Pdx-1-Cre-ER; Prmt5 //I mice (male mice, $n=8$ for each group) were administered with TAM by gavage at the age of 10 weeks. Islets were isolated from the mice at first week and sixth week post TAM administration. Three mice were euthanized for each of these two different time points. The islets used for doing qPCR and perifusion studies were a pool of three individual mice. $(B, C)$ mRNA level of PRMT5 and insulin in islets isolated from Prmt5/l/ and Pdx1-Cre-ER; Prmt5//I mice at first week post TAM administration. (D) In vitro perifusion studies on the islets isolated at first week post TAM administration. (E, F) mRNA level of Prmt5 and insulin in islets isolated from Prmt5//I and $P d \times 1$-Cre-ER; Prmt5//I mice at sixth week post TAM administration. (G) In vitro perifusion studies on the islets isolated at sixth week post TAM administration. $* P<0.05 ; * * P<0.01$. 
Although we did observe significantly impaired GT, we did not see dramatically reduced fasting insulin level in the Prmt5 KO mice. We predicted that some compensatory mechanisms such as beta cell proliferation may occur in vivo as a response to the reduced insulin release. To test this, we performed histological studies to verify whether beta cell proliferation was induced after Prmt5 excision. The pancreas samples were selected from the mice treated with TAM at variant time (i.e. first, sixth, and ninth post TAM administration) and were fixed to make tissue sections. IF staining results showed that a very clear increasing of Ki67 staining, a biomarker for cell proliferation, on the pancreatic islets from Prmt $5 \mathrm{KO}$ mice treated with TAM (Supplementary Fig. 1). However, the increased Ki67 expression did not occurred immediately following TAM-induced Prmt5 excision in islets, but observed at and after sixth week. The quantification of the percentage of insulin staining area over whole pancreas area on the pancreas tissue sections from the mice at the ninth week post TAM treatment also indicated an increasing beta cell mass in Prmt5 $\mathrm{KO}$ mice relative to control mice (Supplementary Fig. 2). Collectively, the islet-specific conditional KO of Prmt5 leads to IGT and defective GSIS. But the beta cell proliferation we found here seems a result of compensation to impaired insulin release and glucose intolerance when deleted PRMT5 in islets. This compensatory proliferation of beta cell would contribute to antagonize insulin expression decreasemediated IGT.

\section{The islet-specific conditional Prmt5 KO reduced intracellular insulin expression in beta cells}

Several conditions have been implicated in impaired insulin response under glucose stimulation, such as low level of insulin synthesis, inefficiency of insulin secretion, and even non-response to glucose stimulation (Fu et al. 2013). Here, we found that insulin mRNA level was downregulated in mouse islets after Prmt5 excision (Fig. 4C and F). However, whether PRMT5 regulates insulin transcription is not clear. To this end, we investigated the effect of Prmt5 KO in influencing intracellular synthesis of insulin in the mouse islets using QPCR, Western blot, and immunostaining. Notably, qRT-PCR showed a significant decline of insulin mRNA level in mouse islets with Prmt5 KO (Figs 4C and F, 5A). Consistent with reduced insulin mRNA, the proinsulin protein level was also downregulated as compared with the control groups (Fig. 5B, C and D). Further, the insulin IF staining also showed lower insulin expression in the pancreatic islets from Prmt5 $\mathrm{KO}$ mice (Fig. 5E). The quantification with ImageJ showed that the insulin level was moderately, yet significantly lower in Prmt5 KO mice than that in the control mice (Fig. 5F), correlating with the lower mRNA and protein levels in the islets (Fig. 5A, B, C and D). These results indicate that the Prmt5 excision led to reduced insulin expression in islets.

To further confirm these results, the Prmt5 expression was downregulated by specific shRNA in INS- 1 cells. After PRMT5 knockdown (KD), the expression level of insulin
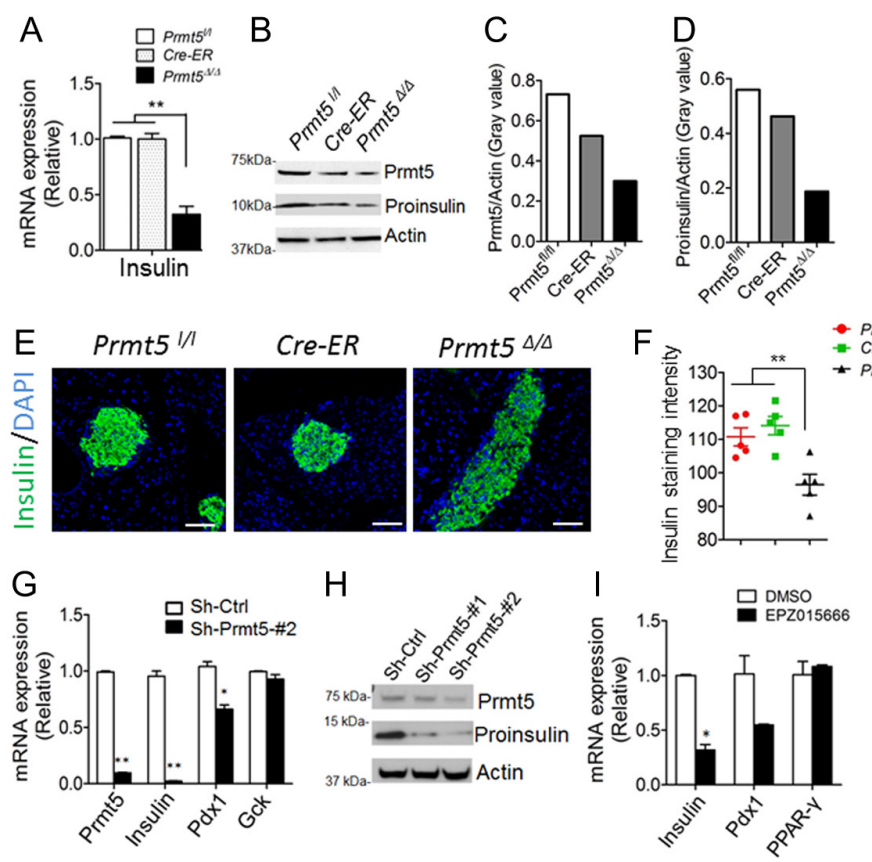

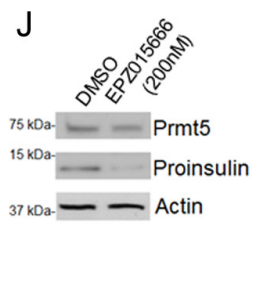

\section{Figure 5}

Islet-specific Prmt5 deletion in mice reduced intracellular insulin level in beta cells. (A) Insulin mRNA level in islets of control and Prmt5 KO mice was determined by qPCR. (B) Western blot for detecting the protein level changes of PRMT5 and proinsulin in islets of Prmt5 $\mathrm{KO}$ mice and control mice. (C, D) Quantification of PR5 and proinsulin expression levels in Western blot assay. (E) Immunostaining for insulin and nucleus (DAPI) (Scale bar, $25 \mu \mathrm{m}$ ) and representative images from control and Prmt5 KO groups. (F) Quantitation and comparison of insulin fluorescence density between control and Prmt5 excision mice groups. It was calculated from the integrated density data of insulin fluorescence signals measured using ImageJ software. (G, H) Prmt5-specific shRNA treatment on INS-1 cells suppresses the intracellular expression of PRMT5 and insulin in mRNA level (qPCR) and protein levels (Western blot). $(I$, J) PRMT5 enzyme activity specific inhibitor EPZ015666 treatment decreases the intracellular mRNA and protein levels of proinsulin in INS-1 cells. $* P<0.05 ; * \star P<0.01$. 
was decreased sharply in the mRNA level (Fig. 5G) and also in protein level (Fig. 5H). Consistent with these findings, treating the INS- 1 cells by a small molecular compound, EPZ015666, a specific inhibitor of PRMT5 enzyme activity (Duncan et al. 2016, Jin et al. 2016, Scaglione et al. 2018), also caused a reduced expression of insulin at both the mRNA level (Fig. 5I) and protein level (Fig. 5J). These results suggest that the PRMT5 deletion or inhibition in islets reduced insulin synthesis.

\section{PRMT5 binds to the promoter of insulin gene and correlates with dimethylation of target histones and binding of an ATP-dependent chromatin remodeling enzyme}

It is well known that PRMT5 functions in transcriptional repression (Karkhanis et al. 2011, Tae et al. 2011). However, some previous reports also suggested that PRMT5 works as a cofactor to activate gene transcription, and this effect is closely linked to the methylation of histone $\mathrm{H} 3$ on Arg8 and chromatin remodeling (Dacwag et al. 2007, LeBlanc et al. 2012). To test how PRMT5 upregulates the insulin gene transcription, we performed chromatin immunoprecipitation (ChIP) with control or PRMT5 specific shRNA-treated INS-1 cell lines. The results showed that PRMT5, dimethylated H3R8, and BRG1 were enriched at the insulin gene promoter in INS-1 cells treated with control-shRNA (Fig. 6A). In contrast, in Prmt5 KD cells, the bindings of PRMT5 and BRG1, as well as the level of dimethylated H3R8 at the insulin promoter were significantly reduced (Fig. 6A). Likewise, knockdown of PRMT5 in INS-1 cells led to the significant reduction of polymerase II (Pol II) binding, which is crucial for gene transcription (Fig. 6B). As a control, PRMT5 KD did not significantly affect the level of total histone 3 level at the insulin gene promoter (Fig. 6C). These results indicate that PRMT5 binds to the promoter of the insulin gene, and this binding is crucial for demethylation of H3R8 and BRG1 binding to the insulin promoter. It is likely that PRMT5 directly promotes insulin gene transcription by dimethylating H3R8 and regulating the binding level of ATP-dependent chromatin remodeling enzymes (Fig. 6D), as PRMT5 promotes gene transcription involved in adipogenic differentiation or skeletal muscle differentiation (Dacwag et al. 2007, LeBlanc et al. 2012).

In addition to the direct regulation on insulin gene by PRMT5, we also examined the impact of Prmt5 excision on transcription level of some insulin secretion-related genes in mouse islets. The results indicated that Prmt5 excision downregulated gene transcription level of $P d x 1$
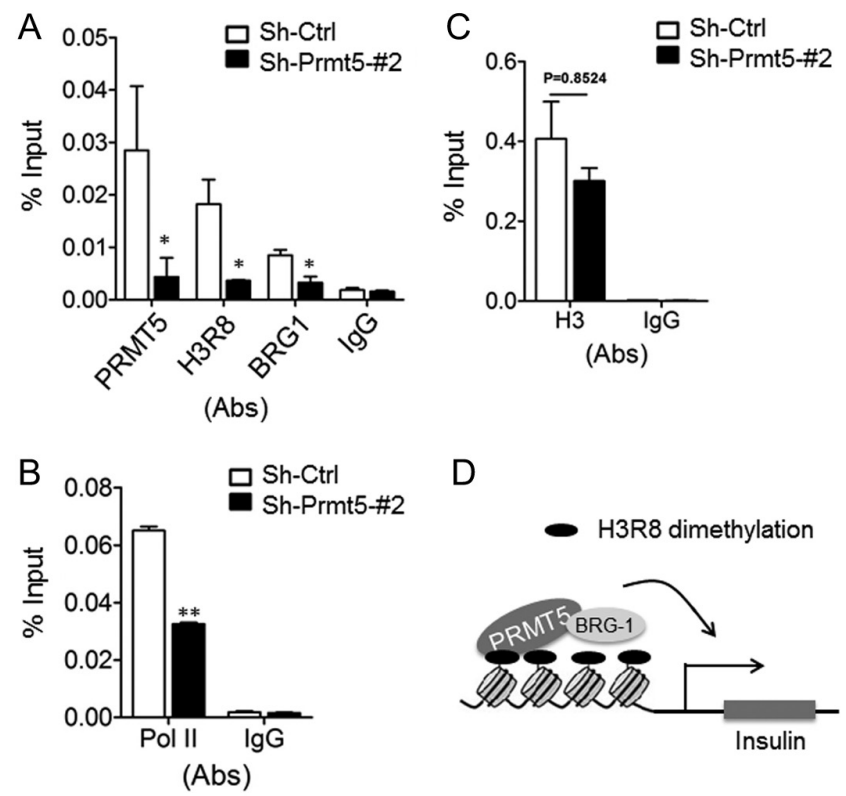

Figure 6

PRMT5 binds to the insulin promoter, and PRMT5 KD reduces dimethylation of H3R8 at the promoter and represses insulin transcription. ChIPs were performed using antibodies against PRMT5, dimethylated (diMe) H3R8, BRG1 and Polymerase II in control and PRMT5 KD INS-1 cell lines treated by Prmt5 specific shRNA. (A) ChIP results demonstrate that binding of PRMT5 and dimethylated H3R8 at the insulin promoter in control and PRMT5 KO INS-1 cells. IgG-binding level to the insulin promoter was used as a negative control. (B) ChIP assay detects Pol II binding at the insulin promoter in control and PRMT5 KD INS-1 cells. (C) Binding of the total histone 3 levels at insulin promoter was found. (D) A suggested model for regulation of insulin transcription by PRMT5 in beta cells. $* P<0.05 ; * * P<0.01$

and Foxa2, but did not influence gene transcription of Gck, Glut2, Sur 1 and Kir 6.2, the genes that affects glucose sensing or insulin secretion (Supplementary Fig. 3). This result implies that some other players, which were also regulated by PRMT5, are possibly involved in impaired insulin secretion besides the directly epigenetic-regulated insulin gene transcription.

\section{Discussion}

Epigenetic regulation plays a crucial role in regulating the homeostasis and function of islets. However, it has been unclear how PRMT5 regulates islets in vivo. Our current studies revealed that targeted Prmt5 excision in islets led to IGT and defective GSIS. Moreover, PRMT5 is essential for optimal insulin expression in vitro and in vivo, and PRMT5 binds the insulin promoter. To our knowledge, this is the first time that definitive evidence showed PRMT5 directly promotes insulin synthesis in beta cells, and PRMT5 is enriched at the insulin promoter. Thus, PRMT5-mediated 
optimal insulin expression may be an important factor that contributes to IGT and defective GSIS in Prmt5 isletsspecific conditional KO mice model.

In current study, $P d x-1$ promoter driven Cre-ER system was used to develop an islet-specific KO of Prmt5. Pdx 1-CreER-containing mice were initially developed by Dr. Melton's group and used to explore the role of Pdx 1 in islets development (Gu et al. 2002). This system was extensively used in our previously published work and shown tissue-specific and TAM-induced Men1 deletion in islets of adult mice (Yang et al. 2010). Here, this system also showed an effectively KO of target gene, i.e., Prmt5 in islet. It provided a valid platform to help us explore the functions of Prmt5 particularly in pancreatic islets.

\section{PRMT5 promotes insulin transcription in beta cells}

Although emerging evidence suggests that PRMT5 functions as an oncogene and is well-recognized as a therapeutic target in cancer treatment (Morettin et al. 2015, Zhang et al. 2015, Tarighat et al. 2016, Yap et al. 2017), its role in regulating metabolism in vivo is not well defined (Tsai et al. 2013). PRMT5 was found to stimulate CREB phosphorylation via increases in histone H3R2 methylation that enhanced chromatin accessibility at gluconeogenic promoters in hepatocytes (Tsai et al. 2013). A recent study also shows that dysfunction of PRMT5 in mice with HFD-induced hepatic steatosis upregulates the expression of the master transcription factors, PPAR $\alpha$ and PGC- $1 \alpha$, which are involved in fatty acid metabolism and mitochondrial biogenesis (Huang et al. 2018). We successfully knocked out the Prmt5 gene in mouse islets and observed the IGT and defective GSIS response in mice. These findings indicate that PRMT5 is involved in regulating glucose metabolism in vivo, at least partly via regulating the synthesis of insulin in islets beta cells.

To investigate the mechanism underlying the PRMT5-mediated regulation of insulin transcription, we found PRMT5, dimethylated H3R8, and BRG1 enriched at the promoter of insulin using ChIP assay. Notably, Prmt5 KO led to the reduction of the amount of PRMT5, dimethylated H3R8 and BAG1 at the promoter. These findings indicate that PRMT5 may directly promote insulin gene transcription by dimethylating H3R8 and increase the binding level of BRG1, a component of an ATP-dependent chromatin remodeling enzyme, to insulin promoter to remodel chromatin structure there. This is consistent with previous reports that PRMT5 activated genes transcription which involved in adipogenic differentiation or skeletal muscle differentiation
(Dacwag et al. 2007, LeBlanc et al. 2012). However, more detail mechanism should be elucidated in further experiments to support PRMT5 epigenetically regulating insulin transcription in pancreatic beta cells.

Besides the direct regulation of insulin transcription by PRMT5, it is well known that the insulin mRNA is also impacted by various diabetogenic stressors, for example, glucotoxicity, oxidative/ER stress, and inflammation. Here, as we observed an obvious IGT in Prmt5 KO mice, the decreased insulin mRNA level may be one result of the IGT. However, about 50\% decline of the insulin expression level in islets isolated at the first week post TAM treatment help us to exclude that IGT is a cause to the downregulated insulin transcription in Prmt5 $\mathrm{KO}$ mice, as the IGT in Prmt5 KO mice just took place at the sixth week after TAM administration, but the reduction of insulin expression occurred within 1 week following Prmt5 deletion (Fig. 4). Nevertheless, it remains to be further investigated on how PRMT5 deletion leads to IGT.

\section{Prmt5 KO influences expression of the insulin secretion-related genes}

In addition to inadequate synthesis of insulin, reduced uptake of glucose and impaired insulin secretion are also involved in reduced insulin secretion after glucose stimulation (Fu et al. 2013). Previous studies have shown that glucose sensors including glucose transporter 2 (GLUT2) and glucokinase (GCK) play crucial roles in response to blood glucose uptake and utilization (Janssen et al. 1994, Thorens 2015). As to the regulation of insulin secretion, the priming and fusion of insulin-containing granules precede insulin exocytosis and secretion. This event is triggered by elevation of intracellular $\left[\mathrm{Ca}^{2+}\right]$ (Gauthier \& Wollheim 2008, Ashcroft \& Rorsman 2012). Sulfonylurea receptor1 (SUR1) was involved in the regulation of $\mathrm{Ca}^{2+}$-induced exocytosis by interacting with a $\mathrm{Ca}^{2+}$ sensor, Piccolo (Fujimoto et al. 2002) and a cAMP-binding protein called CAMPS (Ozaki et al. 2000). Previous studies also showed that the ATP-sensitive potassium channel (KATP channel) is an essential ion channel involved in glucose-induced insulin secretion. The deletion of the components of KATP channel, SUR 1 and KIR6.2, will lead to a defective GT (Winarto et al. 2001). In addition, FOXA2 has been identified as an essential activator of genes that function in multiple pathways governing insulin secretion (Lantz et al. 2004, Wolfrum et al. 2004).

Our results show that Prmt5 KO did not reduce the expression of GLUT2 and GCK in islets. The transcription 
factors that upregulate GLUT2 and GCK expression, PDX1 and PPAR- $\gamma$ (Im et al. 2006), was not significantly changed when downregulated PRMT5 expression. These results suggest that Prmt5 KO may not cause IGT and defective GSIS via influencing glucose uptake and phosphorylation by glucose kinase in beta cells. Nevertheless, we could not rule out the possibility that Prmt5 deletion influences insulin secretion through regulating other processes of beta cell biology such as ion-channel-regulated insulin secretion, even though our results show that Prmt5 KO did not downregulate the Kir6.2 and Sur-1 gene (Supplementary Fig. 3). And further studies are also required to determine whether Prmt5 KO regulated FOXA2 expression influences insulin secretion as we found that the Foxa 2 gene mRNA level was reduced after Prmt5 KO (Supplementary Fig. 3).

\section{Beta cell proliferation following Prmt5 excision may be a compensatory mechanism to control blood glucose level against impaired insulin synthesis}

As Prmt5 KO led to significant reduction of insulin synthesis in beta cells, marked IGT and quite low fasting insulin level should be observed in Prmt5 KO mice. In this study, we did observe significantly IGT, but we did not see dramatically reduced fasting insulin level in the Prmt5 KO mice. In this regard, we found that Prmt5 excision resulted in a gradually increased proliferation of beta cells in mouse islets. In our previous study, we found that PRMT5 interacts with menin to epigenetically suppress Hedgehog signaling by imparting the repressive H4R3m2s mark at the Gas1 promoter (Gurung et al. 2013). It suggests that PRMT5 is a suppressor to beta cell proliferation. In the current study, our in vivo data did not show an immediately proliferation of beta cell after PRMT5 excision, rather a gradual and late increase in proliferation following development of IGT. The qPCR data did not show that PRMT5 deletion increases the expression level of Gas-1 in islets as observed in the insulinoma cells (Gurung et al. 2013) (Supplementary Fig. 3). Thus, the beta cell proliferation after Prmt5 KO in mouse islets seems a compensatory response to defective insulin secretion. On the other hand, we cannot rule out the possibility that acute Prmt5 excision may lead gradual and slow epigenetic changes in chromatin that results in enhanced beta cell proliferation.

In addition, some studies recently proved an inverse relationship between beta cell proliferation and function (Klochendler et al. 2016, Puri et al. 2018). The data we obtained in this study, that is, the early inhibition of insulin transcription and late happen of beta cell proliferation in Prmt5 KO mice, may help us rule out the possibility for that the defective insulin secretion is a direct result of beta cell proliferation.

Collectively, we evaluated the effect of PRMT5 deletion in beta cells on beta cell function in vivo. Slight increase in fasting blood glucose level and markedly IGT in mice with Prmt5 excision in islets were observed. This phenotype may reflect a compensatory outcome of enhanced beta cell numbers as a result of deficient insulin synthesis in Prmt5 KO mice. The net effect of Prmt5 KO may explain that the Prmt5 KO mice showed IGT and insufficient GSIS due to reduced insulin synthesis in beta cells; yet, the relatively normal fasting blood insulin level resulting from the increased islet mass to maintain background level of insulin. Given the important effect of PRMT5 on insulin synthesis by these studies, these findings will enhance the understanding of beta cell biology. Moreover, these results will likely provide new insights into exploring novel strategies to treat diabetes caused by insulin insufficiency.

\section{Supplementary materials}

This is linked to the online version of the paper at https://doi.org/10.1530/ JOE-19-0268.

\section{Declaration of interest}

The authors declare that there is no conflict of interest that could be perceived as prejudicing the impartiality of the research reported.

\section{Funding}

This work was supported by National Institutes of Health (NIH) (grant numbers: 1-R01-CA-178856 and R01 DK097555), a Harrington Discovery Institute Innovator Scholar Award, and a Sanofi Innovation Award (iAward).

\section{Author contribution statement}

$\mathrm{J} M, \mathrm{XH}$, and $\mathrm{XH}$ conceived and designed the studies, and performed the analysis and interpretation of the data.J $\mathrm{M}, \mathrm{XH}, \mathrm{YC}, \mathrm{KO}, \mathrm{KM} \mathrm{S}, \mathrm{YW}, \mathrm{B} \mathrm{G}, \mathrm{ZF}$, and $\mathrm{B}$ W K performed the experiments. J M and $\mathrm{XH}$ drafted the manuscript and all other authors revised it. All authors approved the final version of the paper.

\section{Acknowledgements}

The authors are grateful for the processing of samples for histological studies at the Molecular Pathology and Imaging Core at the University of Pennsylvania, and for the isolation of mouse islets at the Pancreatic Islet Cell Biology Core at the University of Pennsylvania. (c) 2019 Society for Endocrinology Published by Bioscientifica Ltd. Printed in Great Britain 


\section{References}

Antonioli L, Blandizzi C, Csoka B, Pacher P \& Hasko G 2015 Adenosine signalling in diabetes mellitus - pathophysiology and therapeutic considerations. Nature Reviews Endocrinology 11 228-241. (https://doi. org/10.1038/nrendo.2015.10)

Ashcroft FM \& Rorsman P 2012 Diabetes mellitus and the beta cell: the last ten years. Cell 148 1160-1171. (https://doi.org/10.1016/j. cell.2012.02.010)

Bedford MT \& Clarke SG 2009 Protein arginine methylation in mammals: who, what, and why. Molecular Cell 33 1-13. (https://doi. org/10.1016/j.molcel.2008.12.013)

Bernstein D, Golson ML \& Kaestner KH 2017 Epigenetic control of betacell function and failure. Diabetes Research and Clinical Practice 123 24-36. (https://doi.org/10.1016/j.diabres.2016.11.009)

Chen H, Gu X, Su IH, Bottino R, Contreras JL, Tarakhovsky A \& Kim SK 2009 Polycomb protein Ezh2 regulates pancreatic beta-cell Ink4a/ Arf expression and regeneration in diabetes mellitus. Genes and Development 23 975-985. (https://doi.org/10.1101/gad.1742509)

Dacwag CS, Ohkawa Y, Pal S, Sif S \& Imbalzano AN 2007 The protein arginine methyltransferase Prmt5 is required for myogenesis because it facilitates ATP-dependent chromatin remodeling. Molecular and Cellular Biology 27 384-394. (https://doi.org/10.1128/MCB.01528-06)

DeFronzo RA, Ferrannini E, Groop L, Henry RR, Herman WH, Holst JJ, Hu FB, Kahn CR, Raz I, Shulman GI, et al. 2015 Type 2 diabetes mellitus. Nature Reviews Disease Primers 1 15019. (https://doi. org/10.1038/nrdp.2015.19)

Dhawan S, Tschen SI, Zeng C, Guo T, Hebrok M, Matveyenko A \& Bhushan A 2015 DNA methylation directs functional maturation of pancreatic beta cells. Journal of Clinical Investigation 125 2851-2860. (https://doi.org/10.1172/JCI79956)

Duncan KW, Rioux N, Boriack-Sjodin PA, Munchhof MJ, Reiter LA, Majer CR, Jin L, Johnston LD, Chan-Penebre E, Kuplast KG, et al. 2016 Structure and property guided design in the identification of PRMT5 tool compound EPZ015666. ACS Medicinal Chemistry Letters 7 162-166. (https://doi.org/10.1021/acsmedchemlett.5b00380)

Feng Z, Ma J \& Hua X 2017a Epigenetic regulation by the menin pathway. Endocrine-Related Cancer 24 T147-T159. (https://doi. org/10.1530/ERC-17-0298)

Feng Z, Wang L, Sun Y, Jiang Z, Domsic J, An C, Xing B, Tian J, Liu X, Metz DC, et al. 2017b Menin and Daxx interact to suppress neuroendocrine tumors through epigenetic control of the membrane metallo-endopeptidase. Cancer Research 77 401-411. (https://doi. org/10.1158/0008-5472.CAN-16-1567)

Fernandez-Zapico ME, van Velkinburgh JC, Gutierrez-Aguilar R, Neve B, Froguel P, Urrutia R \& Stein R 2009 MODY7 gene, KLF11, is a novel p300-dependent regulator of Pdx-1 (MODY4) transcription in pancreatic islet beta cells. Journal of Biological Chemistry 28436482 36490. (https://doi.org/10.1074/jbc.M109.028852)

Ferrannini E \& Mari A 2014 beta-Cell function in type 2 diabetes. Metabolism: Clinical and Experimental 63 1217-1227. (https://doi. org/10.1016/j.metabol.2014.05.012)

Fu Z, Gilbert ER \& Liu D 2013 Regulation of insulin synthesis and secretion and pancreatic Beta-cell dysfunction in diabetes. Current Diabetes Reviews 9 25-53. (https://doi. org/10.2174/157339913804143225)

Fujimoto K, Shibasaki T, Yokoi N, Kashima Y, Matsumoto M, Sasaki T, Tajima N, Iwanaga T \& Seino S 2002 Piccolo, a Ca2+ sensor in pancreatic beta-cells. Involvement of cAMP-GEFII.Rim2. Piccolo complex in cAMP-dependent exocytosis. Journal of Biological Chemistry 277 50497-50502. (https://doi.org/10.1074/jbc.M210146200)

Gauthier BR \& Wollheim CB 2008 Synaptotagmins bind calcium to release insulin. American Journal of Physiology: Endocrinology and Metabolism 295 E1279-E1286. (https://doi.org/10.1152/ ajpendo.90568.2008)
Girardot M, Hirasawa R, Kacem S, Fritsch L, Pontis J, Kota SK, Filipponi D, Fabbrizio E, Sardet C, Lohmann F, et al. 2014 PRMT5-mediated histone $\mathrm{H} 4$ arginine-3 symmetrical dimethylation marks chromatin at G+C-rich regions of the mouse genome. Nucleic Acids Research $\mathbf{4 2}$ 235-248. (https://doi.org/10.1093/nar/gkt884)

Gu G, Dubauskaite J \& Melton DA 2002 Direct evidence for the pancreatic lineage: NGN3+ cells are islet progenitors and are distinct from duct progenitors. Development 129 2447-2457.

Gurung B, Feng Z, Iwamoto DV, Thiel A, Jin G, Fan CM, Ng JM, Curran T \& Hua X 2013 Menin epigenetically represses Hedgehog signaling in MEN1 tumor syndrome. Cancer Research 73 2650-2658. (https://doi. org/10.1158/0008-5472.CAN-12-3158)

Guz Y, Montminy MR, Stein R, Leonard J, Gamer LW, Wright CV \& Teitelman G 1995 Expression of murine STF-1, a putative insulin gene transcription factor, in beta cells of pancreas, duodenal epithelium and pancreatic exocrine and endocrine progenitors during ontogeny. Development 121 11-18.

Huang L, Liu J, Zhang XO, Sibley K, Najjar SM, Lee MM \& Wu Q 2018 Inhibition of protein arginine methyltransferase 5 enhances hepatic mitochondrial biogenesis. Journal of Biological Chemistry 29310884 10894. (https://doi.org/10.1074/jbc.RA118.002377)

Im SS, Kim SY, Kim HI \& Ahn YH 2006 Transcriptional regulation of glucose sensors in pancreatic beta cells and liver. Current Diabetes Reviews 2 11-18. (https://doi.org/10.2174/157339906775473581)

Janssen RC, Bogardus C, Takeda J, Knowler WC \& Thompson DB 1994 Linkage analysis of acute insulin secretion with GLUT2 and glucokinase in Pima Indians and the identification of a missense mutation in GLUT2. Diabetes 43 558-563. (https://doi.org/10.2337/ diab.43.4.558)

Jin Y, Zhou J, Xu F, Jin B, Cui L, Wang Y, Du X, Li J, Li P, Ren R, et al. 2016 Targeting methyltransferase PRMT5 eliminates leukemia stem cells in chronic myelogenous leukemia. Journal of Clinical Investigation 126 3961-3980. (https://doi.org/10.1172/JCI85239)

Karkhanis V, Hu YJ, Baiocchi RA, Imbalzano AN \& Sif S 2011 Versatility of PRMT5-induced methylation in growth control and development. Trends in Biochemical Sciences 36 633-641. (https://doi.org/10.1016/j. tibs.2011.09.001)

Klochendler A, Caspi I, Corem N, Moran M, Friedlich O, Elgavish S, Nevo Y, Helman A, Glaser B, Eden A, et al. 2016 The genetic program of pancreatic beta-cell replication in vivo. Diabetes 65 2081-2093. (https://doi.org/10.2337/db16-0003)

Kong X, Yan D, Sun J, Wu X, Mulder H, Hua X \& Ma X 2014 Glucagonlike peptide 1 stimulates insulin secretion via inhibiting RhoA/ROCK signaling and disassembling glucotoxicity-induced stress fibers. Endocrinology 155 4676-4685. (https://doi.org/10.1210/en.20141314)

Lantz KA, Vatamaniuk MZ, Brestelli JE, Friedman JR, Matschinsky FM \& Kaestner KH 2004 Foxa2 regulates multiple pathways of insulin secretion. Journal of Clinical Investigation 114 512-520. (https://doi. org/10.1172/JCI21149)

Le Guezennec X, Vermeulen M, Brinkman AB, Hoeijmakers WA, Cohen A, Lasonder E \& Stunnenberg HG 2006 MBD2/NuRD and MBD3/NuRD, two distinct complexes with different biochemical and functional properties. Molecular and Cellular Biology 26 843-851. (https://doi.org/10.1128/MCB.26.3.843-851.2006)

LeBlanc SE, Konda S, Wu Q, Hu YJ, Oslowski CM, Sif S \& Imbalzano AN 2012 Protein arginine methyltransferase 5 (Prmt5) promotes gene expression of peroxisome proliferator-activated receptor $\gamma 2$ (PPAR $\gamma 2)$ and its target genes during adipogenesis. Molecular Endocrinology 26 583-597. (https://doi.org/10.1210/me.2011-1162)

Matkar S, Thiel A \& Hua X 2013 Menin: a scaffold protein that controls gene expression and cell signaling. Trends in Biochemical Sciences $\mathbf{3 8}$ 394-402. (https://doi.org/10.1016/j.tibs.2013.05.005)

Matkar S, Sharma P, Gao S, Gurung B, Katona BW, Liao J, Muhammad AB, Kong XC, Wang L, Jin G, et al. 2015 An epigenetic pathway regulates sensitivity of breast cancer cells to HER2 inhibition via https://joe.bioscientifica.com

https://doi.org/10.1530/JOE-19-0268
C 2019 Society for Endocrinology Published by Bioscientifica Ltd. Printed in Great Britain 
FOXO/c-Myc Axis. Cancer Cell 28 472-485. (https://doi.org/10.1016/j. ccell.2015.09.005)

Menzies KJ, Zhang H, Katsyuba E \& Auwerx J 2016 Protein acetylation in metabolism - metabolites and cofactors. Nature Reviews Endocrinology 12 43-60. (https://doi.org/10.1038/nrendo.2015.181)

Miller CP, McGehee RE, Jr \& Habener JF 1994 IDX-1: a new homeodomain transcription factor expressed in rat pancreatic islets and duodenum that transactivates the somatostatin gene. $E M B O$ Journal 13 1145-1156. (https://doi.org/10.1002/j.1460-2075.1994. tb06363.x)

Morettin A, Baldwin RM \& Cote J 2015 Arginine methyltransferases as novel therapeutic targets for breast cancer. Mutagenesis 30 177-189. (https://doi.org/10.1093/mutage/geu039)

Olson LK, Redmon JB, Towle HC \& Robertson RP 1993 Chronic exposure of HIT cells to high glucose concentrations paradoxically decreases insulin gene transcription and alters binding of insulin gene regulatory protein. Journal of Clinical Investigation 92 514-519. (https://doi.org/10.1172/JCI116596)

Ozaki N, Shibasaki T, Kashima Y, Miki T, Takahashi K, Ueno H, Sunaga Y, Yano H, Matsuura Y, Iwanaga T, et al. 2000 cAMP-GEFII is a direct target of cAMP in regulated exocytosis. Nature Cell Biology 2 805-811. (https://doi.org/10.1038/35041046)

Pal S, Yun R, Datta A, Lacomis L, Erdjument-Bromage H, Kumar J, Tempst P \& Sif S 2003 mSin3A/histone deacetylase 2- and PRMT5containing Brg1 complex is involved in transcriptional repression of the Myc target gene cad. Molecular and Cellular Biology 23 7475-7487. (https://doi.org/10.1128/mcb.23.21.7475-7487.2003)

Pal S, Baiocchi RA, Byrd JC, Grever MR, Jacob ST \& Sif S 2007 Low levels of miR-92b/96 induce PRMT5 translation and H3R8/H4R3 methylation in mantle cell lymphoma. EMBO Journal 26 3558-3569. (https://doi.org/10.1038/sj.emboj.7601794)

Papizan JB, Singer RA, Tschen SI, Dhawan S, Friel JM, Hipkens SB, Magnuson MA, Bhushan A \& Sussel L 2011 Nkx2.2 repressor complex regulates islet beta-cell specification and prevents beta-to-alpha-cell reprogramming. Genes and Development 25 2291-2305. (https://doi. org/10.1101/gad.173039.111)

Peers B, Leonard J, Sharma S, Teitelman G \& Montminy MR 1994 Insulin expression in pancreatic islet cells relies on cooperative interactions between the helix loop helix factor E47 and the homeobox factor STF-1. Molecular Endocrinology 8 1798-1806. (https://doi.org/10.1210/ mend.8.12.7708065)

Peshavaria M, Gamer L, Henderson E, Teitelman G, Wright CV \& Stein R 1994 XIHbox 8, an endoderm-specific Xenopus homeodomain protein, is closely related to a mammalian insulin gene transcription factor. Molecular Endocrinology 8 806-816. (https://doi.org/10.1210/ mend.8.6.7935494)

Pinney SE \& Simmons RA 2010 Epigenetic mechanisms in the development of type 2 diabetes. Trends in Endocrinology and Metabolism 21 223-229. (https://doi.org/10.1016/j.tem.2009.10.002)

Puri S, Roy N, Russ HA, Leonhardt L, French EK, Roy R, Bengtsson H, Scott DK, Stewart AF \& Hebrok M 2018 Replication confers beta cell immaturity. Nature Communications 9 485. (https://doi.org/10.1038/ s41467-018-02939-0)

Rank G, Cerruti L, Simpson RJ, Moritz RL, Jane SM \& Zhao Q 2010 Identification of a PRMT5-dependent repressor complex linked to silencing of human fetal globin gene expression. Blood 1161585 1592. (https://doi.org/10.1182/blood-2009-10-251116)

Rui J, Deng S, Lebastchi J, Clark PL, Usmani-Brown S \& Herold KC 2016 Methylation of insulin DNA in response to proinflammatory cytokines during the progression of autoimmune diabetes in NOD mice. Diabetologia 59 1021-1029. (https://doi.org/10.1007/s00125016-3897-4)

Scaglione A, Patzig J, Liang J, Frawley R, Bok J, Mela A, Yattah C, Zhang J, Teo SX, Zhou T, et al. 2018 PRMT5-mediated regulation of developmental myelination. Nature Communications 9 2840. (https:// doi.org/10.1038/s41467-018-04863-9)

Tae S, Karkhanis V, Velasco K, Yaneva M, Erdjument-Bromage H, Tempst P \& Sif S 2011 Bromodomain protein 7 interacts with PRMT5 and PRC2, and is involved in transcriptional repression of their target genes. Nucleic Acids Research 39 5424-5438. (https://doi.org/10.1093/ nar/gkr170)

Tarighat SS, Santhanam R, Frankhouser D, Radomska HS, Lai H, Anghelina M, Wang H, Huang X, Alinari L, Walker A, et al. 2016 The dual epigenetic role of PRMT5 in acute myeloid leukemia: gene activation and repression via histone arginine methylation. Leukemia 30 789-799. (https://doi.org/10.1038/leu.2015.308)

Thorens B 2015 GLUT2, glucose sensing and glucose homeostasis. Diabetologia 58 221-232. (https://doi.org/10.1007/s00125-014-3451-1)

Tsai WW, Niessen S, Goebel N, Yates JR, 3rd, Guccione E \& Montminy M 2013 PRMT5 modulates the metabolic response to fasting signals. PNAS 110 8870-8875. (https://doi.org/10.1073/pnas.1304602110)

Wei H, Mundade R, Lange KC \& Lu T 2014 Protein arginine methylation of non-histone proteins and its role in diseases. Cell Cycle $\mathbf{1 3} 32-41$. (https://doi.org/10.4161/cc.27353)

Winarto A, Miki T, Seino S \& Iwanaga T 2001 Morphological changes in pancreatic islets of KATP channel-deficient mice: the involvement of KATP channels in the survival of insulin cells and the maintenance of islet architecture. Archives of Histology and Cytology 64 59-67. (https:// doi.org/10.1679/aohc.64.59)

Wolfrum C, Asilmaz E, Luca E, Friedman JM \& Stoffel M 2004 Foxa2 regulates lipid metabolism and ketogenesis in the liver during fasting and in diabetes. Nature 432 1027-1032. (https://doi.org/10.1038/ nature03047)

Wu KL, Gannon M, Peshavaria M, Offield MF, Henderson E, Ray M, Marks A, Gamer LW, Wright CV \& Stein R 1997 Hepatocyte nuclear factor 3 beta is involved in pancreatic beta-cell-specific transcription of the pdx-1 gene. Molecular and Cellular Biology 17 6002-6013. (https:// doi.org/10.1128/mcb.17.10.6002)

Yang Y \& Bedford MT 2013 Protein arginine methyltransferases and cancer. Nature Reviews Cancer 13 37-50. (https://doi.org/10.1038/ nrc3409)

Yang Y, Gurung B, Wu T, Wang H, Stoffers DA \& Hua X 2010 Reversal of preexisting hyperglycemia in diabetic mice by acute deletion of the Men1 gene. PNAS 107 20358-20363. (https://doi.org/10.1073/ pnas.1012257107)

Yap TA, Aerts JG, Popat S \& Fennell DA 2017 Novel insights into mesothelioma biology and implications for therapy. Nature Reviews Cancer 17 475-488. (https://doi.org/10.1038/nrc.2017.42)

Zhang B, Dong S, Li Z, Lu L, Zhang S, Chen X, Cen X \& Wu Y 2015 Targeting protein arginine methyltransferase 5 inhibits human hepatocellular carcinoma growth via the downregulation of beta-catenin. Journal of Translational Medicine 13 349. (https://doi. org/10.1186/s12967-015-0721-8)

Zhao Q, Rank G, Tan YT, Li H, Moritz RL, Simpson RJ, Cerruti L, Curtis DJ, Patel DJ, Allis CD, et al. 2009 PRMT5-mediated methylation of histone H4R3 recruits DNMT3A, coupling histone and DNA methylation in gene silencing. Nature Structural and Molecular Biology 16 304-311. (https://doi.org/10.1038/nsmb.1568)

Received in final form 29 August 2019

Accepted 19 September 2019

Accepted Manuscript published online 19 September 2019 https://joe.bioscientifica.com https://doi.org/10.1530/JOE-19-0268 (c) 2019 Society for Endocrinology Published by Bioscientifica Ltd. Printed in Great Britain 